This is a pre-print version of a manuscript that was accepted for publication in the International Journal for Philosophy of Religion. Please, only cite the published version. You can read the official manuscript for free using the following link: https://rdcu.be/csYL1

\title{
An Epistemological Challenge to Ontological Bruteness
}

\begin{abstract}
It is often assumed that the first stage of many classical arguments for theism depends upon some version of the Principle of Sufficient reason (PSR) being true. Unfortunately for classical theists, PSR is a controversial thesis that has come under rather severe criticism in the contemporary literature. In this article I grant, for the sake of argument, that every version of PSR is false. Thus, I concede with the critics of PSR that there is, at least, one fact that is ontologically brute. I then proffer an epistemological challenge to ontological bruteness. Specifically, I argue that whenever a knowledge seeker, S, perceives some unexplained fact, F, it is never reasonable for $\mathrm{S}$ to believe $\mathrm{F}$ is ontologically brute. I conclude that, even if PSR is false, it is more reasonable to believe that the key premise in the first stage of classical arguments for theism is true.
\end{abstract}

Keywords: Brute Facts; Explanations; Classical Theism; the Principle of Sufficient Reason; Epistemology

\section{Introduction}

The first stage of many classical arguments for the existence of God can be formulated as follows:

\section{Classical Arguments for Theism: Stage One (CAT-1)}

1.1. Contingent fact, $\mathrm{F}$, obtains

1.2. If $\mathrm{F}$ obtains, then $\mathrm{F}$ has an explanation, $\mathrm{E}$, for why it obtains.

1.3. Therefore, $\mathrm{F}$ has an explanation, $\mathrm{E}$, for why it obtains. ${ }^{1}$

Depending on which particular classical argument one is concerned with, ' $\mathrm{F}$ ' may be any number of contingent facts. For example, ' $F$ ' may be the fact that the universe is ordered, or the fact that the universe undergoes change, or the fact that the universe is a composite entity, and so on and so forth. ${ }^{2}$ In Stage Two of such arguments, further reasons are typically provided to show that 'E'

\footnotetext{
${ }^{1}$ The classical arguments for theism I have in mind are plentiful in the ancient literature. For some examples see the works of Philo of Alexandria (1993 originally, 20-50BC p.537), Maximos the Confessor (2014, originally, 628-30 pp. 3-5), and John of Damascus (1958, originally, 743 pp.168-70). Additionally, Lloyd Gerson, (1993) and (1994), has done an unparalleled job reconstructing Neoplatonic arguments for theism, such as one finds in the likes of Plotinus. The Jewish and Christian thinkers I just cited were heavily influenced by Middle Platonic and Neoplatonic philosophy.

${ }^{2}$ The arguments develop in this article do not depend on one being committed to a facts ontology. If one wanted to, she could reformulate CAT-1 so as to avoid talk about facts. For example, one could restate 1.2. as follows: $<$ If there is an $x$ such that $x$ instantiates $y$, there is a $z$ such that $z$ fully or partially explains why $x$ instantiates $y>$ where ' $x$ ' is 'the universe' and ' $y$ ' is some relation or property like 'being ordered', 'being composite', or 'being beautiful'. I, however, find it more convenient to talk about facts.
} 
This is a pre-print version of a manuscript that was accepted for publication in the International Journal for Philosophy of Religion. Please, only cite the published version. You can read the official manuscript for free using the following link: https://rdcu.be/csYL1

corresponds to the God of Classical Theism. In this article, however, we shall only be concerned with CAT-1.

Typically, a classical theist takes 1.1. to be such an obvious truth that it is virtually undeniable. ${ }^{3}$ Premise 1.2., on the other hand, is often believed to be justified on the basis of some version of the Principle of Sufficient Reason (PSR). Roughly, one who is committed to the truth of PSR either believes there are no ontologically brute facts or that there are no ontologically brute facts within a particular subset of facts; for example, many defenders of PSR maintain there are no ontologically brute contingent facts. Given the truth of PSR, one can argue that 1.2 is true because, necessarily, $\mathrm{F}$ has an explanation, $\mathrm{E}$, for why it obtains.

Since many theists appeal to PSR to support premise 1.2, it is commonly assumed that the success of CAT-1 depends on some version of PSR being true. This is problematic because, unfortunately for defenders of theism, PSR is a controversial principle that has recently come under rather severe criticism. ${ }^{4}$ In the face of such criticisms, it behooves theists to consider other supporting arguments for the second premise of CAT-1. In this article, I do this by proffering an epistemological challenge to ontological bruteness.

Notably, I grant, for the sake of argument, that every version of PSR is false. Thus, I concede with critics of PSR that it is possible there is, at least, one contingent fact that is ontologically brute. Nevertheless, I argue that whenever a knowledge seeker, S, perceives some unexplained fact, $\mathrm{F}$, it is never reasonable for $\mathrm{S}$ to believe $\mathrm{F}$ is ontologically brute. Thus, $\mathrm{I}$ conclude that it is always more reasonable to believe 1.2. is true than false. I begin by laying the groundwork for my argument-focusing on several crucial preliminary issues. I clarify what I mean by an 'explanation' and an 'unexplained fact'. I also clarify the distinction between 'epistemological bruteness' versus 'ontological bruteness'. Following this, I outline and defend my argument that it is never reasonable for a knowledge seeker to believe an unexplained fact is ontologically brute.

\footnotetext{
${ }^{3}$ Bertrand Russell famously proposed always, "to begin any argument that I have to make by appealing to data which will be quite ludicrously obvious" (Russell, 1940 p.6). In a similar manner, classical theists traditionally begin arguments for theism by appealing to data that might be considered "ludicrously obvious" or incorrigible and, thus, not in need of any additional justification.

${ }^{4}$ Perhaps the most devastating contemporary criticism of PSR has been proffered by Peter van Inwagen (1983; 2009). There are, however, other objections to PSR. For a detailed survey of these objections, as well as responses to them, see Pruss (2006). Recently, to circumvent the formidable challenge of van Inwagen's objection to PSR, its defenders have reformulated the principle in terms of metaphysical grounding; e.g., see Dasgupta (2016) and Amijee (2020). In response to this trend, McDaniel (2020) has pushed back and argued that, even if one formulates PSR in terms of grounding, the principle is subject to a relevantly similar version of van Iwagen's powerful objection.
} 
This is a pre-print version of a manuscript that was accepted for publication in the International Journal for Philosophy of Religion. Please, only cite the published version. You can read the official manuscript for free using the following link: https://rdcu.be/csYL1

\section{Explanations}

For any unexplained fact, $\mathrm{F}$, at least one of three things may be true:

I. F has an objective explanation and it is epistemically possible for someone to come to know F's explanation.

II. F has an objective explanation and it is epistemically impossible for someone to come to know F's explanation.

III. F does not have an objective explanation.

I maintain that whenever a knowledge seeker, $\mathrm{S}$, perceives an unexplained fact, $\mathrm{F}$, it may be reasonable for $\mathrm{S}$ to believe I or II is true; but it is never reasonable for $\mathrm{S}$ to believe III is true. Before developing this argument, however, there are a few crucial preliminary issues I need to focus on. To begin with I need to provide an analysis of the concept of an 'explanation' and clarify what it means to say that a fact is unexplained. I also need to explain the difference between epistemological and ontological bruteness. Once I have clarified these terms, I will be prepared to explicate my argument.

Let us begin by considering the concept of an 'explanation'. I take it that an explanation is a proposition put forth to account for some explanandum, i.e., some obtaining fact. An explanation is, thus, an answer to a why or in virtue of what question regarding some fact or obtaining state-of-affairs like the following:

Q1: 'Why or in virtue of what does some fact, F, obtain?'5

Tentatively, therefore, we can define an explanation as follows:

Explanation: A proposition, $\mathrm{E}$, is an explanation of some fact $\mathrm{F}$ iff $\mathrm{E}$ provides a positive answer to Q1.

Note that in order to count as an explanation E must provide a positive answer to Q1. One could, of course, respond to Q1 negatively by saying 'I don't know' or 'Nothing explains why F obtains' but these are hardly explanations. To understand this better consider another example. Here is a paradigmatic example of a fact that requires an explanation regarding why it obtains:

F1: Barack Obama is the first African American president of the United States.

\footnotetext{
${ }^{5}$ Here I am following Bas van Fraassen's (1980) contention that explanations are answers to why questions. It is important to note, however, that Jaworski (2009) has argued, quite persuasively, that explanations also encompass answers to how questions. While this is an important point, the distinction between why and how questions is not directly relevant to the topic at hand. Thus, for the sake of concision and clarity I have chosen to focus exclusively on why questions.
} 
This is a pre-print version of a manuscript that was accepted for publication in the International Journal for Philosophy of Religion. Please, only cite the published version. You can read the official manuscript for free using the following link: https://rdcu.be/csYL1

One might ask the following question: 'Why or in virtue of what does F1 obtain?'. Presumably, the person asking this does so because she is seeking knowledge regarding the circumstances involving presidential elections and the first African American being elected president. If one responded by saying 'I don't know why F1 obtains' or 'Nothing explains why F1 obtains' then the knowledge seeker asking the question hasn't come to know anything about why Barack Obama is the first African American president. This highlights an important point. Explanations are supposed to impart knowledge regarding why or in virtue of what some fact obtains. Hence, negative responses to Q1 do not count as explanations.

Let us now consider an explanation one might plausibly put forth for why F1 obtains:

E1: F1 obtains because: (i) Barack Obama exemplifies the property 'being an African American', (ii) all previous American presidents exemplify the property 'being Caucasian', and (iii) in 2008 Barack Obama secured 365 Electoral College votes and nearly 53 percent of the popular vote, thus, winning the presidential election.

As E1 illustrates, there can be multiple answers to any given question. In other words, one can proffer multiple different propositions in answer to questions of type Q1; this entails that some explanations are only partial explanations. In E1, the truth of (i), for example, only partially explains why F1 obtains. A complete explanation requires the truth of (ii) and (iii) as well (and probably some other important propositions that I have missed out). For simplicity sake, however, let us tentatively stipulate that E1 is a complete explanation. If E1 is a complete explanation, and one is justified in believing it is true, then one is said to know F1's explanation, i.e., to know why or in virtue of what F1 obtains.

Furthermore, the various explanations put forth to explain why some fact, like F1, obtains can track with or correspond to multiple different facts composed of different types of objects, properties, and relations. So, for example, if (i) is true then it corresponds to a state-of-affairs composed of the person, Barack Obama, exemplifying the property 'being an African American'. Likewise, if (iii) is true then it corresponds to a fact composed of the events in 2008 involving Barack Obama winning the Electoral College and popular votes. It is the conjunction of these different obtaining facts or states-of-affairs (and probably some others I have failed to mention here) that, together, provide a complete explanation for F1.

Okay, now I have outlined a general account of what I mean by an explanation. This account of explanations is, however, too coarse-grained. To properly develop the argument that it is never reasonable for a knowledge seeker to believe III is true, I need to develop a slightly more fine-grained account of explanations. It is to this that I will now turn my attention to.

As should be clear by now some (perhaps most) facts require multiple explanations regarding why they obtain if one is searching for a complete explanation. In addition to this, however, it is also important to note that there are multiple different ways one can interpret Q1. I take it that the various ways one might interpret Q1 correspond to the different types of explanations one might put forth in response to Q1. Let me unpack what I mean by this. When one poses a question like Q1, she usually means something more specific like Q1 ${ }^{\alpha}$ : 
This is a pre-print version of a manuscript that was accepted for publication in the International Journal for Philosophy of Religion. Please, only cite the published version. You can read the official manuscript for free using the following link: https://rdcu.be/csYL1

\section{Q1 ${ }^{\alpha}$ : 'What caused some fact $\mathrm{F}$ to obtain?}

One who interprets Q1 as Q1 ${ }^{\alpha}$ is searching for a causal explanation. In other words, she expects a full or partial explanation to track with or correspond to the events that brought about the fact that F obtains. To better understand this, consider, again, F1.

Suppose that when I posed the question, 'Why or on account of what does F1 obtain?', what I had in mind was 'What caused or brought about the fact that F1 obtains?'. Given this, one would expect that the answer to this question would include the events that brought about the fact that F1 obtains. Indeed, the plausible explanation, E1, that I offered above reveals that I took F1 to require a causal explanation. E1 includes the events that caused Obama to become president; namely, those involving Obama winning the Electoral College and popular votes in the 2008 presidential election that caused him to win the presidency. as $\mathrm{Q} 1^{\beta}$ :

There is, however, another way one can interpret Q1; namely, Q1 can also be interpreted

\section{Q1 ${ }^{\beta}$ : 'What grounds the fact that F obtains?'}

When one interprets $\mathrm{Q} 1$ as $\mathrm{Q} 1^{\beta}$ she is seeking a grounding explanation. In this particular case, she expects a full or partial answer to track with or correspond to the entity or entities that ground the fact that F obtains. So, returning to our example, when I posed the question, 'Why or on account of what does F1 obtain?', I also could have meant, 'What grounds the fact that F1 obtains?'. Once again, a closer look at E1 reveals I did interpret it in this way. E1 does not merely include the events that caused Obama to become the first African American president, but also the state-of-affairs that grounds the fact that F1 obtains, i.e., that Obama bears the property 'being an African American', and all previous American presidents bear the property 'being Caucasian'.

There is yet another way one could interpret Q1. One could interpret Q1 as Q1 ${ }^{\gamma}$ :

\section{Q1 ${ }^{\gamma}$ : 'For what end or purpose does F obtain?'}

One who interprets Q1 as Q1 ${ }^{\gamma}$ is seeking a teleological explanation. Accordingly, she will expect a full or partial answer to include the reason, end, or purpose for which F obtains. It is, therefore, possible that in asking 'Why or on account of what does F1 obtain?' I could have meant, 'For what end or purpose does F1 obtain?'. Some theists maintain there is an objective answer to this question (even if we do not know what that answer is). This is to say, one might argue that God intentionally created a world in which F1 obtains for a specific reason or purpose. We may never know what that reason is, but there is one. An atheist, on the other hand, may be inclined to believe there is no objective reason, end, or purpose for which F1 obtains. The claim that F1 has an objective teleological explanation is, thus, controversial. 
This is a pre-print version of a manuscript that was accepted for publication in the International Journal for Philosophy of Religion. Please, only cite the published version. You can read the official manuscript for free using the following link: https://rdcu.be/csYL1

There are, however, uncontroversial examples in which questions of type Q $1^{\gamma}$ clearly do have a positive answer. Consider fact F2:

F2: that the set of wooden chairs in the auditorium, C, are arranged in rows of twenty, two inches apart, and all facing east.

If one were to ask, 'Why or for what end or purpose does F2 obtain?', it would be uncontroversial to assume there is an answer to this question. Indeed, one highly plausible explanation is that the chairs in set $\mathrm{C}$ are arranged in rows of twenty, two inches apart, and all facing east to provide the most optimal view of an upcoming talk. The reason to suppose there is an answer to the question at hand is because, on their own, ordinary wooden chairs do not have the power to arrange themselves into any pattern. Rather, wooden chairs are typically arranged into seating patterns by intelligent agents; and, it is widely believed that agents act to achieve ends or goals (i.e., they act purposively).

The discussion above reveals that, there are times when one can provide an answer to one of Q1's iterations (e.g., Q1 ${ }^{\beta}$ ) but not another (e.g., Q1 ${ }^{\gamma}$ ). Let's return to the example of the hypothetical atheist above. She might argue that facts like F1 are objectively meaningless; namely, that facts like F1 don't obtain for any objective end or purpose. For an atheist who holds this view, there simply is no answer to the question 'For what end or purpose does F1 obtain?'. However, and this is very important, just because she believes there is no objective teleological explanation for F1, it doesn't follow that she believes there is no answer to the question 'Why or in virtue of what does F1 obtain?'. Indeed, our hypothetical atheist may believe E1 provides an adequate explanation regarding why F1 obtains; even though E1 does not include a teleological explanation. This highlights an important point. For a proposition to count as an explanation, it need not answer every possible iteration of Q1. As long as it provides a positive answer to, at least, one iteration of Q1 it counts as being an explanation.

Given all that I have just said regarding the different ways one might interpret Q1, we can refine our definition of an explanation as follows:

Explanation*: A proposition, $\mathrm{E}$, is an explanation of some fact $\mathrm{F}$ iff $\mathrm{E}$ provides a positive answer to at least one fine-grained interpretation of Q1 (e.g., $1^{\alpha}, \mathrm{Q1}^{\beta}, \mathrm{Q}^{\gamma}, \ldots$ ).

A brief disclaimer is now in order. I am sure I have not covered every possible way of interpreting questions of type Q1. My aim, however, was not to provide a complete account of every possible iteration of Q1. There may be other ways of interpreting Q1 that I have failed to mention. ${ }^{6}$ Fortunately, this is not problematic because my goal was merely to show that there are multiple ways in which one can interpret why questions and, hence, different ways of explaining why some fact, F, obtains.

\footnotetext{
${ }^{6}$ By way of example, among philosophers of science, there has recently been an explosion of interest in 'noncausal' scientific explanations; see Lange (2016) and Reutlinger and Saatsi (2018). Even just a brief perusal through this material reveals that there are many different ways of interpreting Q1 that I have not covered here.
} 
This is a pre-print version of a manuscript that was accepted for publication in the International Journal for Philosophy of Religion. Please, only cite the published version. You can read the official manuscript for free using the following link: https://rdcu.be/csYL1

Let us, now, shift our attention to considering what I mean when I say that some fact, F, is unexplained.

\section{Unexplained facts}

On my account, to say that some fact, $\mathrm{F}$, is unexplained, is merely to say that some knowledge seeker (or set of knowledge seekers), S, currently does not know the answer to Q1, i.e., 'Why or in virtue of what does F obtain?'. To be more precise, given 'Explanation*', it means $\mathrm{S}$ doesn't know the answer to any possible interpretation of Q1 (e.g., Q1 ${ }^{\alpha}, \mathrm{Q} 1^{\beta}, \mathrm{Q} 1^{\gamma}$, etc.). Hence, on my account, to say that some fact, $\mathrm{F}$, is unexplained does not entail $\mathrm{F}$ lacks an explanation; nor does it entail there is no one out there who knows F's explanation. Rather, it merely entails that at least one or more knowledge seekers do not know why or in virtue of what F obtains. To better understand what I am claiming, consider the following example. Suppose that an astrophysicist named Gertrude told you the following fact is an unexplained fact:

\section{F3: 'Roughly $68 \%$ of the universe is dark energy'}

On my account, what Gertrude is claiming is that human beings currently do not know the answer to the question 'Why or in virtue of what F3 obtains?' (or to any possible iteration of this question). ${ }^{7}$

For the sake of the example, I will stipulate, further, that Gertrude believes F3 is an unexplained fact because all the best explanations currently proposed are demonstrably false. I will also add that her belief that F3 is an unexplained fact is highly defeasible. Given some new arguments or information Gertrude may modify her belief that F3 is an unexplained fact. For example, suppose that in a year's time, and in light of new observations and testing, her distinguished colleague Johannes convinces her that his partial naturalistic explanation regarding why F3 obtains is true (for the sake of simplicity, let's call this hypothetical explanation E2). Given that Gertrude now believes E2 is true, and that she is justified or warranted in her belief, it would be proper to say that she has a partial explanation for why or in virtue of what F3 obtains. In which case, it would be improper for her to continue claiming that F3 is an unexplained fact. I say all of this to demonstrate that, on my account, an unexplained fact is not one that objectively lacks an explanation. Instead, to say that a given fact, $\mathrm{F}$, is unexplained is ultimately to describe the epistemic state of some knowledge seeker(s). Specifically, it is to say that if a given knowledge seeker (or set of knowledge seekers), S, encounters some fact, F, and does not know the answer to Q1 (or any of its possible interpretations), then S will, rightly, take F to be an unexplained fact. However, S may change her belief if/when she comes to know the answer to at least, one interpretation of Q1.

I must emphasize this last point. According to my account, it is no longer proper for $\mathrm{S}$ to designate $\mathrm{F}$ as being an unexplained fact once she has come to know the answer to one or more

\footnotetext{
${ }^{7}$ I realize physicists are exploring various theories that supposedly explain why F3 obtains and that, perhaps, one of these theories provides the best explanation. My point was not to argue that F3 is actually an unexplained fact. Rather, I am simply using this as a hypothetical example to explain what I mean when I say that some fact is unexplained.
} 
This is a pre-print version of a manuscript that was accepted for publication in the International Journal for Philosophy of Religion. Please, only cite the published version. You can read the official manuscript for free using the following link: https://rdcu.be/csYL1

interpretations of Q1. It only takes coming to know the answer to one iteration of Q1 for a knowledge seeker, S, to say that she knows (or, at least, partially knows) why F obtains. Now that I have explained the unexplainable (Dad joke intended), I am finally in a position to explicate the distinction between epistemological bruteness versus ontological bruteness. ${ }^{8}$ It is to this task that I now turn.

\section{Epistemological versus ontological bruteness}

As I have already stated, whenever one perceives some unexplained fact, F, one of three things may be true. The first is that:

I. F has an objective explanation, and it is epistemically possible for someone to come to know F's explanation.

If $\mathbf{I}$ is true, this entails there exists some fact - composed of some entities (e.g., objects, properties, relations, etc.) - that explains why or in virtue of what F obtains. Given enough time (and the removal of any obstacles that may currently be hindering us), we will eventually come to know the answer to Q1 - or, rather, to one of the fine-grained iterations of

$\mathrm{Q} 1$ like $\mathrm{Q} 1^{\alpha}, \mathrm{Q} 1^{\beta}, \mathrm{Q} 1^{\gamma}$, etc. Note that the moment one does come to know F's explanation, it is no longer correct to say that $\mathrm{F}$ is an unexplained fact. Now, let us consider option two:

II. F has an objective explanation, and it is epistemically impossible for someone to come to know F's explanation.

According to II, F has an explanation, but due to some insurmountable obstacle-e.g., some cognitive or nomological limitation - it is epistemically impossible to come to know what that explanation is. Hence, no amount of time will ever lead to someone coming to know F's explanation. So, like I, II maintains that F has an objective explanation that we currently do not know. In other words, there exists some fact - composed of some entities (e.g., objects, properties, relations, etc.) - that explains why or in virtue of what F obtains. Unlike I, however, II asserts that it is in principle impossible for us to come to know F's explanation. Hence, it is impossible for a knowledge seeker to come to know anything about the state-of-affairs that explains why F obtains.

To get your head around this let us return to the example of the imaginary astrophysicist Gertrude and fact F3. Let us begin by making another stipulation. Assume Gertrude is a proponent of scientism according to which science is the only reliable source of knowledge. Hence, the only types of explanations she will accept are naturalistic/scientific ones. In other words, she doesn't believe F3 has any other type of explanation (e.g., a metaphysical or

\footnotetext{
${ }^{8}$ The distinction between epistemological and ontological brute facts was first introduced by Eric Barnes (1994) and was more recently reiterated by Fahrbach (2005) and Vintiadis and Mekios (2018). My explication of the distinction closely resembles theirs. It is important to note, however, that Elanor Taylor (2018) has challenged this distinction. Taylor claims facts that have an objective explanation that we will never know are not epistemically brute; but, rather, somewhere in between an epistemically and ontologically brute fact. It is not clear to me, however, what she means by this.
} 
This is a pre-print version of a manuscript that was accepted for publication in the International Journal for Philosophy of Religion. Please, only cite the published version. You can read the official manuscript for free using the following link: https://rdcu.be/csYL1

teleological explanation) because such explanations are not derived from scientific methods. In addition to this, let us suppose (hypothetically) that to explain why or in virtue of what F3 obtains she would have to build an observational device the size of a large galaxy. ${ }^{9}$

In this scenario, Gertrude is faced with an insurmountable obstacle that is preventing her from coming to know why F3 obtains. She is cognitively hindered because, without the assistance of the galaxy-sized observational device, she is unable to come to know why F3 obtains. She is also nomologically hindered because it is physically impossible to construct a device the size of a large galaxy. In this hypothetical scenario, the explanandum (i.e., the fact that Roughly $68 \%$ of the universe is dark energy) has an explanans. Unfortunately, it is epistemically impossible to know what it is. Unlike I, II is concerned with facts that a knowledge seeker will never come to know the explanation for. Not because $F$ lacks an objective explanation; but, rather, because there are insurmountable obstacles to coming to know F's explanation.

I and II are both concerned with epistemically brute facts. We can define epistemological bruteness as follows:

Epistemological bruteness: For any fact, F, F is epistemologically brute iff $\mathrm{F}$ has an objective explanation and some knowledge seeker (or set of seekers) S does not know F's explanation.

I must emphasize that an epistemically brute fact objectively has an explanation. The only problem is, we currently do not know F's explanation (and, in some cases, we may never know). I will now turn my attention to the contrasting concept of ontological bruteness.

Whenever we encounter some unexplained fact, $\mathrm{F}$, it is also possible that III is true:

III. F does not have an objective explanation.

Ontologically brute facts are facts that have no objective explanation. They remain permanently unexplained, not because we need more time or technology, or some creative genius, to explain them, or because it is epistemically impossible to explain them, but because such facts simply lack any objective explanation. In other words, there simply is no positive answer to any interpretation of $\mathrm{Q} 1$ (e.g., there is no answer to $\mathrm{Q} 1^{\alpha}, \mathrm{Q} 1^{\beta}, \mathrm{Q} 1^{\gamma}$, etc.). There simply is no state-of-affairs - i.e., no collection of objects, or properties, or relations of any type - that we can point to that make it the case that $\mathrm{F}$ obtains. We can state this more precisely with the following definition:

Ontological bruteness: For any fact, F, F is ontologically brute iff there is no state-ofaffairs that makes it the case that F obtains, and thus F has no objective explanation, i.e.,

\footnotetext{
${ }^{99}$ I want to emphasize that I am not claiming we actually need to build such a device to discover why F3 obtains. This is just a hypothetical scenario.
} 
This is a pre-print version of a manuscript that was accepted for publication in the International Journal for Philosophy of Religion. Please, only cite the published version. You can read the official manuscript for free using the following link: $\underline{\text { https://rdcu.be/csYL1 }}$

there is no positive answer to any possible fine-grained interpretation of Q1 (e.g., Q1 ${ }^{\alpha}$, $\left.\mathrm{Q} 1^{\beta}, \mathrm{Q} 1^{\gamma}, \ldots\right)$.

Now that I have clarified all my terms, I will argue for the conclusion that it is never reasonable to believe that an unexplained fact, F, is ontologically brute. In other words, I will show that it is never reasonable for a knowledge seeker to believe III is true.

\section{An epistemological challenge}

Before I outline my argument, it is important to reiterate an important point. I want to stress, again, that I am not arguing for PSR. PSR is a metaphysical principle. It either claims that for every obtaining fact $\mathrm{F}$, there exists some state-of-affairs that makes it the case that $\mathrm{F}$ obtains; or, it claims that for some subset of obtaining facts, F, there exists some state-of-affairs(s) that makes it the case that every member of $\mathrm{F}$ obtains. In contrast, I am making an epistemological argument, not a metaphysical one. I am arguing that when faced with some unexplained fact, F, it is never reasonable for a knowledge seeker to believe III is true rather than I or II. Thus, on my account, some facts may be ontologically brute even though it is never reasonable for a knowledge seeker to believe that any given unexplained fact she perceives is ontologically brute.

Having made this important clarification, we can finally explicate the argument at hand. Our reasoning can be represented as follows:

\section{An Epistemological Challenge to Ontological Bruteness (ECOB)}

2.1. S perceives an unexplained fact.

2.2. If $\mathrm{S}$ perceives an unexplained fact, it is more reasonable for $\mathrm{S}$ to partially believe that $\mathbf{I}$ is true, unless she encounters a defeater for her partial belief. ${ }^{10}$

2.3. Therefore, it is more reasonable for $\mathrm{S}$ to partially believe that $\mathbf{I}$ is true unless she encounters a defeater for her partial belief.

2.4. Any defeater for S's partial belief that I is true will show that it is more reasonable for $\mathrm{S}$ to believe that II is true or that III is true.

2.5. It is never more reasonable to believe that III is true.

2.6. Therefore, any defeater for S's partial belief that I is true will show that it is more reasonable to believe that II is true.

I shall begin by addressing premises 2.1 and 2.2. It is important to remember that the subject in question, ' $\mathrm{S}$ ', is a knowledge seeker. In this context, this means that $\mathrm{S}$ is a person who both

\footnotetext{
${ }^{10}$ I have opted to use the term 'partial belief' roughly to suggest that there can be more fine-grained attitudes towards propositions aside from all-out belief. Specifically, I take it that one can hold that a given proposition is true with varying degrees of confidence. Presumably, one who partial believes that some proposition, $\mathrm{P}$, is true is more confident in the proposition being true than someone who completely lacks a belief that P. I should add that if one preferred to use the term 'credence' she could. I suspect that someone far more clever than I could develop a Bayesian style argument that assigned a numerical value to the subjective probability that $\mathbf{I}$ is true given humanities overall success at discovering the explanations for unexplained facts. I would also like to note that in using the term 'partial belief' I am not making any commitments regarding the ontological status of 'partial beliefs'; for an excellent survey of the ontological discussion surrounding credence and belief see Jackson (2020).
} 
This is a pre-print version of a manuscript that was accepted for publication in the International Journal for Philosophy of Religion. Please, only cite the published version. You can read the official manuscript for free using the following link: https://rdcu.be/csYL1

desires to know and is motivated to come to know the explanations for unexplained facts. Given that $\mathrm{S}$ is a knowledge seeker, it is relatively easy to make the case that premise 2.2. is true. For, it stands to reason that when a knowledge seeker perceives an unexplained fact, $\mathrm{F}$, she will both desire to know F's explanation and be motivated to come to know F's explanation. Accordingly, S's default position will always be that I is likely to be true, i.e., that it is more probable that F has an explanation and that it is epistemically possible for $\mathrm{S}$ to come to know F's explanation. ${ }^{11}$ If, in contrast, S initially presupposed II or III is true, she would not be motivated to seek out any scientific or philosophical explanation for F. In other words, she would simply give up on seeking out explanations for unexplained facts. This would, effectively, undermine S's status as a knowledge seeker because she would never have a desire to or be motivated to come to know the explanations for unexplained facts. The reason being that $\mathrm{S}$ would always assume that it is either impossible to come to know F's explanation or that F completely lacked an explanation. Furthermore, if every S adopted a default position that II or III is true, this would effectively undermine all philosophical and scientific inquiry. Pragmatically speaking, therefore, the most reasonable assumption for any knowledge seeker to hold initially is that I is likely true until such time that she encounters a defeater for this partial belief.

One might push back and ask whether there is any good reason for S to partially believe that I is true? My response is that, yes, there is. For thousands of years human beings have proffered explanations for unexplained facts and a great many of these explanations are true. In light of this, it is more reasonable for S's default position to be that $\mathrm{F}$ probably has an explanation. Unless one is a proponent of some form of radical skepticism, it just seems straightforwardly more reasonable for $S$ to initially expect that, as with the innumerable unexplained facts that human beings have encountered in the past, $\mathrm{F}$ has an explanation, and it is epistemically possible to come to know what it is. ${ }^{12}$ So, given all that I have just said, we have good reason to accept the conclusion in premise 2.3.

Now, let us shift our attention to premise 2.4. According to 2.4., any defeater for S's partial belief in I will show that it is more reasonable to believe that II or III is true. In other words, any defeater for S's partial belief in I will either make it more reasonable for S to believe that: (i) F has an explanation for why F obtains and she will never know what it is, or (ii) F completely lacks an explanation for why it obtains.

Let us consider the first half of the disjunction in premise 2.4. Might it be reasonable for $\mathrm{S}$ to shift from believing I is true to believing that II is true? Given the right circumstances, I think it may be. To see that this is true, let us consider, once more, the imaginary renowned

\footnotetext{
${ }^{11}$ Some philosophers may prefer to talk about S's attitude towards I in terms of acceptance instead of in terms of degrees of belief. Such a philosopher could simply restate premise 2.2. as follows: <If S perceives an unexplained fact, it is more reasonable for $\mathrm{S}$ to accept that $\mathbf{I}$ is true, unless she encounters a defeater for a belief that $\mathbf{I}$ is true $>$. I do not think this makes a significant difference to what I am arguing in this article. For some foundational discussions about the distinction between 'acceptance' versus 'belief' see Fraassen (1980) and Harmon (1986).

${ }^{12}$ As I suggested in the footnote above, should one feel inclined, she could assign a numerical value to the subjective probability that I is true given the overwhelming success that human beings have had at discovering the explanations for unexplained facts; in which case, she could argue that, initially, S has a high level of credence that I is true.
} 
This is a pre-print version of a manuscript that was accepted for publication in the International Journal for Philosophy of Religion. Please, only cite the published version. You can read the official manuscript for free using the following link: https://rdcu.be/csYL1

astrophysicist Gertrude to illustrate this point. Recall that Gertrude is a proponent of scientism. According to scientism, science alone provides the only viable pathway to knowledge. This entails the only meaningful or important way of interpreting Q1 is one that interprets Q1 as seeking some type of naturalistic or scientific explanation. Assuming Gertrude has good reasons for adopting scientism, she would seem to be justified in her belief that the only viable type of explanation for why F3 obtains is a scientific one. Additionally, given the truth of scientism, Gertrude is right to believe that if F3 has a metaphysical, teleological, or other non-naturalistic explanation, it is epistemically impossible for her to come to know what any of these nonnaturalistic explanations are. The reason being one simply cannot come to know non-naturalistic explanations by means of the scientific method.

I have now set up an example in which $\mathrm{S}$ has a defeater for her partial belief that I is true; one in which it would be reasonable for her to shift from believing I is true to believing II is true. When Gertrude initially encountered F3 she, rightly, held a partial belief that I is true - namely, that F3 had an explanation (in this case a naturalistic one) and that it was epistemically possible for her to come to know what it is. Her confidence in the truth of this assumption, however, soon began to weaken. For, as I stipulated above, after extensive research, Gertrude came to believe that all the best scientific explanations for F3 are false. She later learned that in order to explain why or in virtue of what F3 obtains she would need to build an observational device the size of a large galaxy. In this scenario, as I explained, Gertrude faces insurmountable obstacles for coming to know F3's explanation. Given her scientism, the only type of explanations she can, in principle, come to know are ones that are derived from science. Unfortunately for Gertrude, the only scientific means to finding out why F3 obtains is to build a special device the size of a large galaxy (which is nomologically impossible). This hypothetical scenario shows that if certain conditions obtain it can be reasonable for a knowledge seeker to abandon her initial belief that I is probably true and shift to believing II is true. In the imaginary scenario above, if we grant that Gertrude has good reason to embrace scientism, and if we grant the insurmountable cognitive and nomological obstacles described, we can agree that it is reasonable for her to believe F3 has an explanation but that it is epistemically impossible to come to know what it is. In a moment, we shall see there are other circumstances that may also justify $\mathrm{S}$ believing that II is true as well.

The critical question facing us now, however, is: 'When, if ever, is it more reasonable to shift from believing Ior II is true to believing III is true?'. Put differently: 'When faced with some unexplained fact, $F$, is it ever more reasonable to believe III is true rather than Ior II?'. Clearly, in the scenario above, it is reasonable for Gertrude to believe it is epistemically impossible to come to know F3's explanation. What would it take to make it more reasonable for her to shift to believing the much stronger claim that F3 has no objective explanation? In what follows I argue that there simply are no compelling reasons for Gertrude (or any subject) to make this shift. In other words, I will show that premise 2.5 is true; it is never more reasonable to believe that III is true.

To support premise 2.5. we will consider each of the various reasons $\mathrm{S}$ might proffer for believing III is true. Directly following each reason we will provide a brief response that shows that, at best, the reason in question may give $\mathrm{S}$ a reason to believe that II is true; none of them, however, make it more reasonable to believe that III is true. 
This is a pre-print version of a manuscript that was accepted for publication in the International Journal for Philosophy of Religion. Please, only cite the published version. You can read the official manuscript for free using the following link: https://rdcu.be/csYL1

Reason One: We currently do not know F's explanation. When faced with some unexplained fact, F, S might argue it is reasonable to believe III is true if there is currently no adequate or compelling answer to Q1 (i.e., 'Why or on account of what does $F$ obtain?). In this case, to say there is currently no adequate or compelling answer to Q1 is merely to say that the explanations currently being offered face such serious problems that it is not more reasonable to believe they are true than false.

Response: On its own, the mere fact that $\mathrm{S}$ currently lacks an adequate or compelling answer to Q1 is not enough to undercut her initial partial belief that I is true. Unless she has reason to believe otherwise - like Gertrude's commitment to scientism and her discovery that she would need to build a device the size of a galaxy to come to know F3's explanation-it is possible that given enough time and the removal of any obstacles, she or some other knowledge seeker will eventually develop an adequate answer to Q1. Perhaps, for example, scientists simply need to develop some new (and unforeseen) technology that will enable $\mathrm{S}$ to explain why $\mathrm{F}$ obtains? Or, perhaps, in the next century, a philosophical genius will develop a compelling philosophical explanation for why F obtains? Indeed, given that scientists and philosophers have successfully developed (and continue to develop) compelling explanations for unexplained facts, it seems very reasonable to expect that, eventually, they will come to develop a compelling explanation for F's obtaining. If S should learn that it is not possible (due to some insurmountable obstacle) to come to know F's explanation, this would make it more reasonable for her to believe II is true than I. However, to shift from II to believing III is true, S would need some additional reason for believing that, unlike other unexplained facts, there is no answer to any iteration of Q1 and, thus, that there is no state-of-affairs that makes it the case that F obtains.

Reason Two. There have already been a sufficient number of failed attempts by knowledge seekers to adequately explain why F obtains. One might argue that, when faced with an unexplained fact $\mathrm{F}$, it would be more reasonable for $\mathrm{S}$ to believe III is true if there have been a sufficient number of failed attempts at producing an adequate or compelling explanation for $\mathrm{F}$. Again, in this context, an adequate or compelling answer to Q1 is merely an explanation that is both coherent and in which we have good reason to believe is true.

Response. There is no non-arbitrary way to determine when there have been a sufficient number of failed attempts by knowledge seekers at explaining F. How many attempts at explaining $\mathrm{F}$ must a scientist or philosopher make before it is rational to believe that $\mathrm{F}$ has no explanation whatsoever? 100 attempts? 1,000 attempts? There is simply no objective way to determine the answer to these questions.

The problem, however, is even more acute. Even if we grant, for the sake of argument, that there is a non-arbitrary way to determine when there have been a sufficient number of failed attempts at answering Q1, this does not give us the conclusion that III is true. It may provide us with reason to shift from believing $\mathbf{I}$ is true to believing $\mathbf{I I}$ is true. For example, one might argue the fact that knowledge seekers have utterly failed - after a sufficient number of attempts - to provide an adequate or compelling explanation for $\mathrm{F}$ is evidence that it is epistemically impossible to come to know F's explanation. If the one making such an argument is successful, 
This is a pre-print version of a manuscript that was accepted for publication in the International Journal for Philosophy of Religion. Please, only cite the published version. You can read the official manuscript for free using the following link: https://rdcu.be/csYL1

she would have given $\mathrm{S}$ a compelling reason to believe that II is true. S would still, however, need some further reason to shift from believing II is true to believing III is true.

Ceteris paribus, the fact that there have been a sufficient number of failed attempts by knowledge seekers to adequately explain why F obtains is compatible with both the truth of II and III. So one might argue that, until S acquires further evidence for believing III is true (and not II), it would be more reasonable for S to withhold judgement, i.e., to adopt agnosticism. Remaining agnostic, in this case, simply means that $\mathrm{S}$ requires more evidence before she can justify believing in either II or III. Note, however, that this does not mean it is more reasonable for S to believe that III is true. Indeed, given that there is not enough evidence to shift to believing II or III it is still more reasonable for $\mathrm{S}$ to continuing believing that I is true until such time that she encounters a defeater for this belief. So, even if we grant that a sufficient number of failed attempts at explaining $\mathrm{F}$ have occurred, $\mathrm{S}$ will still need an additional reason to justify believing III is true (rather than II). On its own, then, Reason Two does not compel us to believe III is true.

Reason Three: $\mathrm{F}$ falls outside of the domain of human inquiry. All the disciplines of science, philosophy, religion, subjective experience, and whatever else one may like to include on this list, are incapable (in principle) of enabling a knowledge seeker, S, come to know F's explanation. In other words, suppose it is in principle impossible to answer Q1 (or any of its possible fine-grained iterations). One might argue that if $\mathrm{F}$ falls outside the domain of human inquiry, $\mathrm{S}$ has a good reason for believing III is true.

Response: On the contrary, Reason Three is compatible with either II or III being true. Certainly, if $\mathrm{F}$ falls outside the domain of human inquiry, it follows that it is epistemically impossible for $\mathrm{S}$ to come to know the answer to Q1 (which is precisely what II states). However, it is also possible that $\mathrm{F}$ falls outside of the domain of human inquiry and that III is true. To make it more reasonable for $\mathrm{S}$ to believe that III is true, however, one would need to make an additional argument to show that $\mathrm{F}$ has no objective explanation at all-i.e., that there exists no collection of objects, properties, relations, or whatever-that in some sense make it the case that F obtains. In other words, one would still need to make a further argument showing that $\mathrm{F}$ is not merely epistemically brute but that F is also ontologically brute (as III states). Hence, in the final analysis, not much follows from Reason Three aside from the fact that it entails F stands outside of the domain of human inquiry. ${ }^{13} \mathrm{On}$ its own it doesn't make it more reasonable to believe II is true; and it certainly does not make it more reasonable to believe III is true.

Reason Four: It is metaphysically impossible for certain types of facts to have an explanation. For example, suppose $\mathrm{F}$ is composed of an entity, $x$, that is well-founded, i.e., that is not grounded in anything more fundamental than itself. Also suppose, hypothetically, that S had a good reason for believing the following existence claim is true:

Hypothetical existence Claim: There exists an $x$ such that $x$ does not depend upon anything ontologically prior to (i.e., more fundamental than) itself for its existence.

\footnotetext{
${ }^{13}$ I'd like to thank an anonymous reviewer for pointing this out to me.
} 
This is a pre-print version of a manuscript that was accepted for publication in the International Journal for Philosophy of Religion. Please, only cite the published version. You can read the official manuscript for free using the following link: https://rdcu.be/csYL1

Let the unexplained fact, F, be the fact that the hypothetical existence claim obtains. One might argue that $\mathrm{F}$ is obviously an ontologically brute fact because it is metaphysically impossible for a fact that consists of a fundamental (or well-founded) entity to have an explanation. Given that there is nothing ontologically prior to $x$, one might argue there can be no grounding explanation regarding why $\mathrm{F}$ obtains because $\mathrm{F}$ consists of an entity, $x$, that is ungrounded. As such, the chain of explanation necessarily stops with the fact that F obtains. In other words, $\mathrm{F}$ is a type of fact that completely lacks an explanation in all possible worlds.

Response: To begin with, explanations that track with or correspond to grounding relations are only one type of possible explanation. There are many other possible metaphysical explanations that correspond to states-of-affairs composed of existential dependence relations, instantiation relations, systemic or functional relations, and so on and so forth. For example, although $x$ lacks a ground, it could be that $x$ existentially depends on itself for its existence. Given that $x$ existentially depends on itself for its existence, $x$ would, indeed, have an explanation - one that tracks with a non-grounding dependence relationship. ${ }^{14}$ Furthermore, there are other possible explanations that may come into play including causal, teleological, or even axiological explanations. All of this suggests it would be very challenging for one to successfully establish the thesis that it is metaphysically impossible for a given unexplained fact to have an explanation.

At best, one may be able to compel $\mathrm{S}$ to believe there is at least one interpretation of Q1 (namely, $\mathrm{Q} 1^{\beta}$ ) that has no answer. However, this does not compel $\mathrm{S}$ to believe that all other possible interpretations of Q1 (i.e., Q1 ${ }^{\alpha}, \mathrm{Q} 1^{\gamma}$, etc.) lack an answer-which is what one would need to do to establish that $\mathrm{F}$ is the type of fact that necessarily lacks an answer. It is, however, highly unlikely that anyone could successfully make such an argument. Here is why. Suppose I successfully argued that $\mathrm{F}$ lacks an answer to $\mathrm{Q} 1^{\alpha}, \mathrm{Q} 1^{\beta}$, and $\mathrm{Q} 1^{\gamma}$ ? It is still possible that there is an answer to $\mathrm{Q}^{\delta}$ or $\mathrm{Q}^{\varepsilon}$ and so on and so forth. The point being, unless we have some reason to believe otherwise, it is more reasonable to hold a partial belief that there exists some unknown entity-i.e., some object, property, or relation - that we have not encountered which makes it the case that facts like F obtain. Call this entity $z$. Perhaps, given enough time (and the removal of any obstacles that may currently be hindering us), we will eventually discover $z$ and, thus, come to know why $\mathrm{F}$ obtains? In other words, even if S encounters compelling arguments that $\mathrm{F}$ lacks an answer to $\mathrm{Q} 1^{\alpha}, \mathrm{Q} 1^{\beta}$, and $\mathrm{Q} 1^{\gamma}$, it would still be reasonable for $\mathrm{S}$ to hold a partial belief that $\mathrm{I}$ is true and to continue her search for an explanation for why F obtains. Should S eventually discover it is in principle impossible to come to know why $\mathrm{F}$ obtains this would, indeed, undercut her partial belief that I is true and compel her to believe II is true. It would, not, however, make it more reasonable for $\mathrm{S}$ to believe III is true. In the final analysis, there do not seem to be any

\footnotetext{
${ }^{14}$ In the second stage to classical arguments for God's existence classical theists develop various arguments which purport to show that the fact in question could not existentially depend on itself for its own existence. For example, a classical theist might argue that the fact that the universe is ordered could not existentially depend on itself for its own existence (I am setting aside the details of such an argument for the sake of brevity). Note, however, that even if one is not persuaded by the arguments proffered by classical theists in stage two this does not entail that the fact that the universe is ordered is an ontologically brute fact. It simply entails that nothing grounds the fact that the universe is ordered.
} 
This is a pre-print version of a manuscript that was accepted for publication in the International Journal for Philosophy of Religion. Please, only cite the published version. You can read the official manuscript for free using the following link: https://rdcu.be/csYL1

compelling reasons to believe that it is metaphysically impossible for a given fact of our experience to have an explanation.

Reason Five: F being an ontologically brute fact is part of a general metaphysical theory with strong abductive support (Taylor, $2018 \mathrm{pp} .41-42$ ). Suppose, for example, that some general metaphysical theory $\mathrm{N}$ includes the truth of the proposition $<\mathrm{F}$ is an ontologically brute fact $>$. Further suppose that, through a process of abductive reasoning, $\mathrm{S}$ is compelled to believe $\mathrm{N}$ is true because it is the best general metaphysical theory available. Since, one can argue, $\mathrm{S}$ has good reason to believe $\mathrm{N}$ is true, she also has good reason to believe III is true because her holding III to be true is contained in N.

Response: Reason Five seems compelling until one asks the following question: 'Why does $\mathrm{N}$ take III to be true?'. One might respond that $\mathrm{N}$ takes III to be true because there is currently no adequate explanation for $\mathrm{F}$. It is important to note that the one making this case is ultimately arguing that III is true on the basis of Reason One. We have already shown, however, that Reason One does not support the truth of III. Alternatively, one might maintain N takes III to be true because there have been a sufficient number of failed attempts by knowledge seekers to adequately explain why $\mathrm{F}$ obtains. In which case, she is ultimately arguing that $\mathrm{N}$ takes III to be true based on Reason Two. Unfortunately, this doesn't work either-for we have shown above that Reason Two does not make it more reasonable to believe III is true (rather than I or II).

To this, one might respond $\mathrm{N}$ takes III to be true because, according to $\mathrm{N}, \mathrm{F}$ falls outside of the domain of human inquiry. Ultimately, however, this is an attempt to justify believing III on the basis of Reason Three; which is problematic because we have shown that Reason Three does not make it more reasonable to believe III is true. On the other hand, one might respond that $\mathrm{N}$ takes III to be true because, according to N, F is composed of an entity, $x$, that is well-founded. This, too, is problematic because it is merely to advocate Reason Four. We have already shown, however, that Reason Four does not make it more reasonable to believe III is true (rather than I or II).

As a last resort, one might respond that $\mathrm{N}$ simply stipulates that $\mathrm{F}$ is ontologically brute (and, thus, declares by fiat that III is true). Such a response, however, is not very helpful. Stipulating that some proposition, $\mathrm{P}$, is true does not make it more reasonable to believe $\mathrm{P}$ is true. Surely, stipulating that $\mathrm{N}$ takes III to be true does not make it more reasonable to believe III is true (even if we think $\mathrm{N}$ is the best metaphysical theory in town). In this case, it seems more reasonable for $\mathrm{S}$ to believe $\mathrm{N}$ is true with one caveat - that $\mathrm{S}$ drop the assumption that III is true. Indeed, it isn't even clear that $\mathrm{N}$ entails III is true because there are no good reasons for believing III is true.

In summary, a closer look at Reason Five reveals it is not as compelling as it might initially seem. If $\mathrm{N}$ takes $\mathrm{F}$ to be explanatorily brute based on Reasons One -Four, this is problematic because we have already shown that they do not make it more reasonable to believe III is true. In which case, Reason Five does not make it more reasonable to believe III is true. Conversely, if $\mathrm{N}$ merely stipulates that $\mathrm{F}$ is ontologically brute (without providing any reasons for why this is the case), this also fails to make it more reasonable to believe III is true. For, 
This is a pre-print version of a manuscript that was accepted for publication in the International Journal for Philosophy of Religion. Please, only cite the published version. You can read the official manuscript for free using the following link: https://rdcu.be/csYL1

simply stipulating that III is true does not make it the case that III is actually true; neither does it make it more reasonable to believe III is true. Furthermore, it just does not seem that the truth of III is a necessary feature of N. It seems that one can believe in N and also believe, say, that II is true. Since there does not appear to be any compelling reasons why N entails III is true, the acceptance of $\mathrm{N}$ does not seem to justify a belief that III is true after all. On its own, therefore, Reason Five does not make it more reasonable to believe III is true.

From all of this it follows that it is more reasonable to believe premise 2.5 -which is clearly the most important premise of our argument - is true. The simple reason for this being that there do not appear to be any compelling reasons for a knowledge seeker to believe that III is true. Given this our second conclusion follows: namely, any defeater for S's partial belief that I is true will show that it is more reasonable to believe that II is true. But II merely justifies $\mathrm{S}$ believing that $\mathrm{F}$ is epistemically brute; it does not justify $\mathrm{S}$ believing that $\mathrm{F}$ is ontologically brute. One may push back and complain that I have overlooked an incredibly compelling reason for believing that III is true. My response to this objection is simply to ask, "What is this incredibly compelling reason for believing that III is true?". It seems to me that I have considered the best reasons one might proffer for believing III is true and found them lacking; but, I am certainly open to being corrected.

\section{Conclusion}

If the epistemological challenge to ontological bruteness I just outlined and defended is sound, it follows that it is always more reasonable to believe 1.2. of CAT-1 is true. Recall that 1.2. asserts the following: < If F obtains, then $F$ has an explanation, E, for why it obtains $>$. Since it is never reasonable to believe that III is true, it would, likewise, not be reasonable to believe that 1.2. is false. For asserting that 1.2. is false is the same as asserting that $F$ is ontologically brute, i.e. that III is true. Having said this, it is important to remember that it is still a matter of debate as to whether it is epistemically possible for us to come to know F's explanation. For example, as I have already noted, an ardent proponent of scientism may argue that it is epistemically impossible to know the explanation for facts like 'the fact that the universe is ordered' or 'the fact that the universe undergoes change' (paradigmatic facts appealed to by classical theists in their arguments). Arguably, the explanations for such facts as these can only be arrived at through philosophical methods such as conceptual analysis (and, not, scientific methodologies). Given that science is the only valid pathway to knowledge (as the proponent of scientism believes) one would be justified in believing that $\mathrm{F}$ has an explanation, $\mathrm{E}$, but that it is epistemically impossible to know what $\mathrm{E}$ is. She would not, however, be justified in believing that $\mathrm{F}$ is ontologically brute.

The conclusion of the matter is this: one does not need to appeal to PSR to support the truth of premise 1.2. of CAT-1. In fact, even if one rejects PSR and accepts that there is at least one ontologically brute fact in the world, it would still be more reasonable to believe that premise 1.2. is true than false. For as I have gone through great lengths to show, whenever a 
This is a pre-print version of a manuscript that was accepted for publication in the International Journal for Philosophy of Religion. Please, only cite the published version. You can read the official manuscript for free using the following link: https://rdcu.be/csYL1

knowledge seeker, $\mathrm{S}$, perceives some unexplained fact, $\mathrm{F}$, it is never reasonable for $\mathrm{S}$ to believe $\mathrm{F}$ is ontologically brute.

\section{References}

Amijee, Fatema (2020). 'Principle of Sufficient Reason'. In Michael J. Raven (ed.), The Routledge Handbook of Metaphysical Grounding. New York: Routledge.

Barnes, Eric (1994). 'Explaining Brute Facts'. PSA: Proceedings of the Biennial Meeting of the Philosophy of Science Association 1994:61-68.

Dasgupta, S. (2016) ‘Metaphysical Rationalism', Nous, 50 (2), 379-418.

Fahrbach, Ludwig (2005). 'Understanding Brute Facts'. Synthese 145 (3):449-466.

Gerson, Lloyd P. (1993). Plotinus's Metaphysics: Emanation or Creation? Review of Metaphysics 46 (3):559 - 574.

, Lloyd P. (1994). Plotinus. Routledge.

Harman, Gilbert (1986). Change in View. MIT Press.

Jackson, Elizabeth G. (2020). The relationship between belief and credence. Philosophy Compass 15 (6):1-13.

Jaworski, William (2009). 'The logic of how-questions'. Synthese 166 (1):133 - 155.

John of Damascus (1958, originally, 743). Writings. Translated and edited by Chase Jr. \& H. Frederic. The Catholic University of America Press.

Lange, Marc (2016). Because Without Cause: Non-Causal Explanations in Science and Mathematics. Oxford University Press.

Maximos the Confessor (2014, originally, 628-30). On Difficulties in the Church Fathers: The Ambigua Volume II. Translated and edited by Nicholas Constas. Harvard University Press.

McDaniel, Kris (2019). 'The principle of sufficient reason and necessitarianism'. Analysis 79 (2):230-236.

Philo of Alexandria (1993, originally, 20-50BC). The Works of Philo: Complete and Unabridged. Translated by Charles Duke Yonge. Hendrickson Publishers.

Pruss, Alexander R. (2006). The Principle of Sufficient Reason: A Reassessment. Cambridge University Press. 
This is a pre-print version of a manuscript that was accepted for publication in the International Journal for Philosophy of Religion. Please, only cite the published version. You can read the official manuscript for free using the following link: https://rdcu.be/csYL1

Reutlinger, Alexander \& Saatsi, Juha (eds.) (2018). Explanation Beyond Causation: Philosophical Perspectives on Non-Causal Explanations. Oxford University Press.

Russell, Bertrand (ed.) (1940). The Philosophy of Logical Atomism. Routledge.

Taylor, Elanor (2018). 'How to make the case for brute facts'. In Elly Vintiadis \& Constantinos Mekios (eds.), Brute Facts. Oxford University Press.

van Fraassen Bas, C. (1980). The Scientific Image. Oxford University Press.

van Inwagen, Peter (1983). An Essay on Free Will. Oxford University Press.

van Inwagen, P. (2009). Metaphysics. Boulder: Westview Press.

Vintiadis, Elly \& Mekios, Constantinos (eds.) (2018). Brute Facts. Oxford University Press. 